\section{Jean Plouët \\ Chargé de recherche au Cnrs.}

\section{Christiane Dorey \\ Ingénieur Inserm}

\section{RÉFÉRENCES}

1. Stryer L. Cyclic GMP cascade of vision. Ann Rev Neurosci $1986 ; 9$ : 87-119.

2. Goridis C, Virnaux N, Cailla HL, Delaage MA. Rapid, light-induced changes of retinal cyclic GMP levels. FEBS Lett 1974; 49 : 167-9.

3. Fesenko EE, Kolesnikov SS, Lyubarsky AL. Induction by cyclic GMP of cationic conductance in plasma membrane of retinal rod outer segment. Nature $1985 ; 313: 310-3$.

4. Yosnikami S, Hagins WA. Control of the dark current in vertebrate rods and cones. In : Langer $\mathrm{H}$, ed. Biochemistry and Physiology of Visual Pigments. New York: Springer-Verlag, 1973 : 245-55.

5. Matthews HR, Torre V, Lamb TD. Effects on the photoresponse of calcium buffers and cyclic GMP incorporated into the cytoplasm of retinal rods. Nature $1985 ; 313$ : 582.5.

6. Puckett KL, Goldin SM. Guanosine 3', 5' cyclic monophosphate stimulates release of actively accumulated calcium in purified disks from rod outer segments of bovine retina. Biochemistry $1986 ; 25: 1739-46$.

\section{ADRESSE}

J. Plouët : Inserm U. 86, laboratoire d'immunopathologie de l' $\propto$ il, centre des Cordeliers, 15, rue de l'École de Médecine, 75270 Paris

\title{
La transduction visuelle
}

Le segment externe des bâtonnets est un exemple extrême de différenciation et de spécialisation ; $90 \%$ des protéines $y$ jouent un rôle dans la transduction visuelle, ce qui a beaucoup facilité l'étude du système. Rhodopsine, transducine, antigène-S, phosphodiestérase à GMP cyclique et canaux à sodium constituent ainsi un modèle privilégié de la perception d'un signal et de sa " transduction" vers les effecteurs cellulaires, modèle dont un nombre croissant de données nouvelles indique qu'il est même un archétype de l'organisation des récepteurs membranaires et des G-protéines.

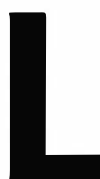

a rétine réagit à la lumière par une réponse électrique adaptée graduellement à son niveau d'éclairement initial. L'ensemble des réactions de conversion d'énergie photonique en impulsion électrique se déroule dans l'extrémité distale, c'est-àdire le segment externe, des cellules photoréceptrices. Celles-ci comprennent les bâtonnets et les cônes, respectivement spécialisés dans la discrimination de l'intensité lumineuse et des couleurs. Le segment externe des bâtonnets constitue un matériel biologique extrêmement précieux pour l'étude d'une fonction différenciée : $90 \%$ environ des protéines totales sont impliquées dans la transduction visuelle. L'unité fonctionnelle est un disque flottant dans le cytoplasme, formé par invagination de la membrane plasmique et constitué essentiellement du photorécepteur (la rhodopsine). La formation des disques se fait au niveau du cil du bâtonnet qui unit le segment externe au segment interne. Ce cil est fragile et sa rupture permet d'isoler les segments externes à l'état pur (figure 1). En revanche, il n'est pas actuellement possible de séparer le segment externe des cônes, et il ne sera pas ici ques- tion de la machinerie transductionnelle assurant la vision des couleurs.

\section{Quel second messager?}

La nature du second messager mis en jeu au cours de la transduction visuelle a été sujette à polémiques durant une dizaine d'années (revue de Stryer [1]). Il est admis à l'heure actuelle que le second messager est la guanosine monophosphate cyclique (GMPc). GMPc : l'élu. Les segments externes contiennent une concentration exceptionnellement élevée de GMPc $(70 \mu \mathrm{M})$ qui diminue sous l'effet d'une phosphodiestérase spécifique activée par la lumière [2]. Un seul photon déclenche l'hydrolyse de $10^{5}$ molécules de GMPc, ce qui est en accord avec l'amplitude du courant d'hyperpolarisation observé. Inversement, quelques millisecondes après une injection intracellulaire de GMPc, la membrane plasmique se dépolarise, contrebalançant l'hyperpolarisation induite par la lumière. En 1985, Fesenko [3] apporta la preuve directe que le GMPc ouvre les canaux sodium. Il a utilisé pour ces expériences des membranes de segments externes dont la face cytoplasmique était orientée vers 
la solution à tester. L'ouverture des canaux se produit en absence de GTP ou d'ATP, ce qui indique que le processus ne dépend pas d'une protéine kinase. L'ouverture est proportionnelle à la concentration du GMPc et n'est pas affectée par l'ion calcium. Par ailleurs, la cinétique de fermeture des canaux est parallèle à la cinétique d'hydrolyse du GMPc par la phosphodiestérase activée par la lumière. L'analyse des flux de GMPc intracytoplasmique est compatible avec la fonction d'adaptation du segment externe. L'hydrolyse du GMPc par la phosphodiestérase activée par la lumière est rapidement compensée par une activation de la guanylate cyclase qui permet une reconstitution rapide des pools de GMPc.

Ion calcium : l'exclu. Il fut d'abord proposé que la lumière provoquait le relargage du calcium de l'espace intradiscal vers le cytoplasme [4], ce qui sembla confirmé par le fait que l'intro- duction de $\mathrm{Ca}^{++}$dans le segment externe induisait la fermeture des canaux sodium. Cette hypothèse fut ensuite infirmée car les mouvements étaient trop lents pour être physiologiquement efficaces, et également parce que des segments externes plongés en milieu sans calcium continuaient de répondre à la stimulation lumineuse [5]. Il semble qu'à l'obscurité le calcium soit accumulé dans le segment externe par passage à travers le canal sodium et relargué par l'échangeur sodiumcalcium. Sous l'effet de la lumière, le canal sodium se ferme, contribuant à majorer le relargage dû à l'échangeur sodium-calcium qui, lui, n'est pas modifié par la lumière. Par ailleurs, la diminution du GMPc entraînerait également une sortie de calcium par un troisième type de mécanisme encore mal caractérisé [6]. Ces phénomènes rendent compte de l'adaptation, mais semblent trop lents pour que le calcium joue efficacement un rôle de second

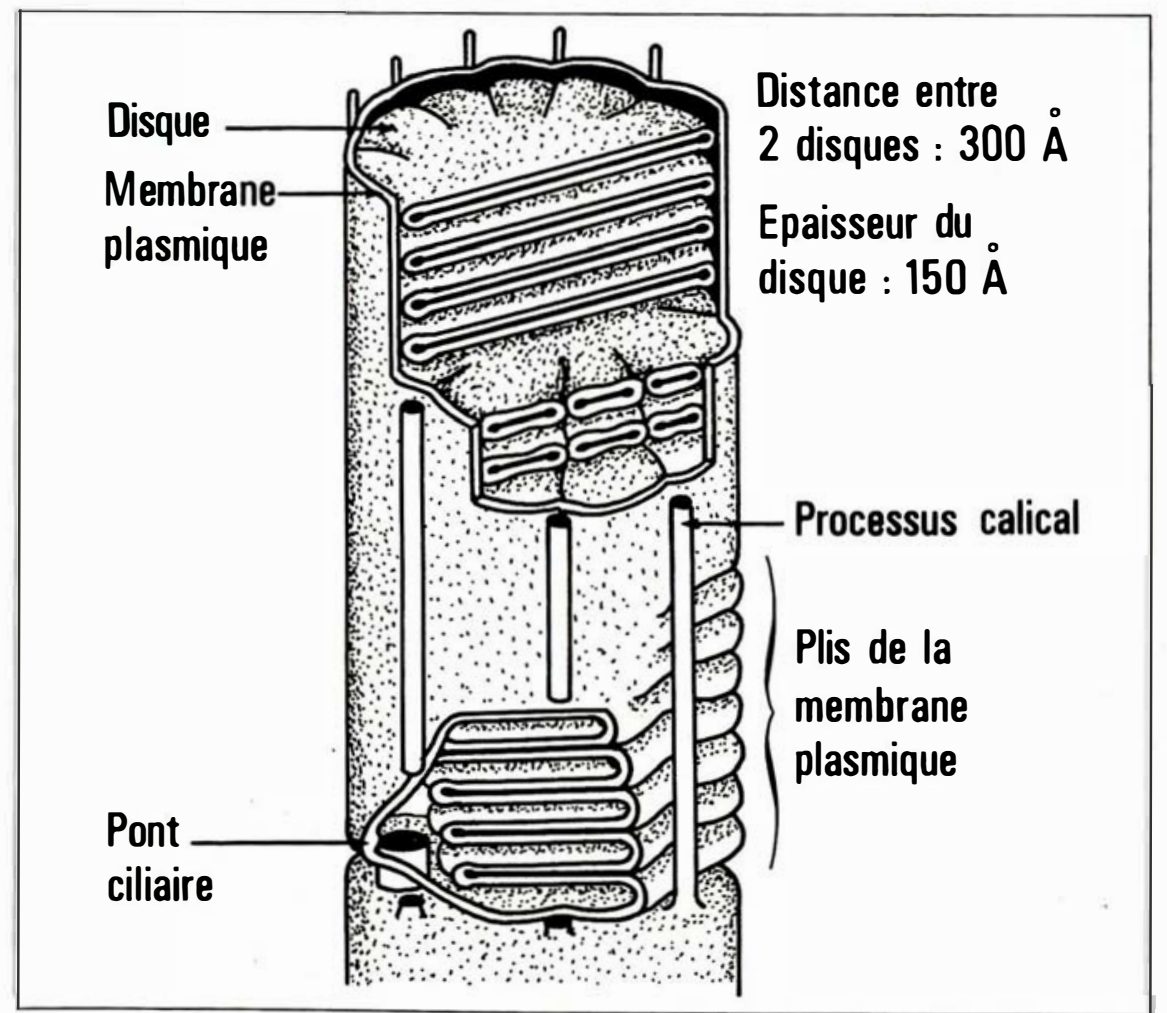

Figure 1. Coupe schématique du segment externe d'un bâtonnet (repris d'après Fein et Szuts). messager [7]. Cette baisse transitoire du calcium intra-cytoplasmique stimule la guanylate cyclase [8] et inhibe la phosphodiestérase, provoquant un rétrocontrôle du second messager, le GMPc. Il semble donc bien que l'ion calcium joue un rôle de modulateur de la transduction visuelle et non de second messager. Inositol triphosphate : un nouveau venu. La lumière provoque l'hydrolyse du phosphatidylinositol 4,5-diphosphate. Par ailleurs, l'injection de 1,4,5 inositol triphosphate (IP3) dans le segment externe de vertébré induit une hyperpolarisation équivalente à celle observée sous l'action de la lumière [9]. En revanche, l'éclairement intermittent diminue l'intensité du courant d'hyperpolarisation produit par IP3 ; ce courant recouvre lentement son niveau basal malgré la poursuite de la stimulation par IP3. Cette adaptabilité laisse penser que l'IP3 jouerait un rôle de second messager dans la transduction visuelle.

\section{La cascade d'amplification}

Métarhodopsine II : la photolyse du récepteur. La lumière isomérise le pigment visuel 11 -cis rétinal en tout-trans rétinal, ce qui confere à la rhodopsine de nouvelles propriétés spectroscopiques engendrées en cascade : bathorhodopsine pendant quelques picosecondes, puis lumirhodopsine pendant quelques nanosecondes, puis métarhodopsine I pendánt quelques microsecondes, puis métarhodopsine II en environ 1 milliseconde. Enfin, la métarhodopsine II s'hydrolyse en quelques minutes en opsine et tout-trans rétinal. Cette cascade de photolyse existe spontanément dans l'obscurité à un taux d'environ $10^{23}$ fois moindre qu'à la lumière, ce qui confere une remarquable stabilité au photorécepteur.

Des études spectroscopiques ont montré que la nécessaire amplification du signal photonique s'accomplissait par une interaction protéique au niveau de la métarhodopsine II [10]. 


\section{RÉFÉRENCES}

7. Torre V. Matthews HR, Lamb TD. Role of calcium in regulating the cyclic GMP cascade of phototransduction in retinal rods. Proc Natl Acad Sci USA 1986 ; 83 : 7109-13.

8. Pepe JM, Pandolfi I, Cugnoli. Guanylate cyclase in rod outer segments of the toad retina. FEBS Lett 1986 ; 203 : 73-6.

9. Waloga G, Anderson RE. Effects of inositol-1,4, 5-triphosphate injections into salamander rods. Biochem Biophys Res Commun $1985 ; 126$ : 59-62.

10. Bennet $\mathbf{N}$, Michel-Villaz $\mathbf{M}$, Kuhn $\mathrm{H}$. Light-induced interaction between rhodopsin and the GTP-binding protein. Eur J Biochem 1982 ; $127: 97-103$

11. Kuhn H. Light-and GTP-regulated interaction of GTPase and other proteins with bovine photoreceptor membranes. Nature 1980 ; 283 : 587-9.

12. Baehr W, Devlin MJ, Applebury ML. Isolation and characterization of cGMP phosphodiesterase from bovine rod outer segments. J Biol Chern 1979 ; 254 : 11669-71.

13. Plouët J, Mascarelli F, Lagente O, et al. Eye derived growth factor : a component of rod outer segment implicated in phototransduction. In : Agardh E, Ehinger B, eds. Retinal Signal Systems. Degenerations and Transplants. Amsterdam : Elsevier, $1986 ; 311-20$.

14. Magnaldo I, L'Allemain G, Chambard JC, Moenner M, Barritault D, Pouyssegur J. The mitogenic signaling pathway of fibroblast growth factor is not mediated through polyphosphoinositide hydrolysis and protein kinase C activation in hamster fibroblasts. $J$ Biol Chem 1986 : 261 : 169016-22.

15. Kuhn H. Light-regulated binding of rhodopsin kinase and other proteins to cattle photoreceptor membranes. Biochemistry $1978 ; 17$ : 4389-95.

16. Wilden U, Hall SW, Kuhn H. Phosphodiesterase activation by photoexcited rhodopsin is quenched when rhodopsin is phosphorylated and binds 48 kDa-protein. Proc Natl Acad Sci USA $1986 ; 83$ : 1174-8.

17. Pfister C, Chabre M, Plouët J, et al. Retinal S-antigen identified as the $48 \mathrm{~K}$ protein regulating light-dependent phosphodiesterase in rods. Science $1985 ; 228: 891-3$.

18. Wiltow GJ, Katial A, Craft C, Shinohara $T$. Sequence analysis of bovine retinal $S$ antigen. Relationship with transducin and Gproteins. FEBS Lett 1986 ; 196 : 23-8.

19. Plouët J. Dorey C, Pandolfino MC, Lorans G, Faure JP. Relation activité-structure entre les modulateurs positif-transducine-et négatif- antigène-S- de la phototransduction. Biol Cell 1986 ; 57 a : 51 .

20. Zuckerman R, Cheasty JE. A $48 \mathrm{kDa}$ protein arrests cGMP phosphodiesterase activation in retinal rod disk membranes. FEBS Lett 1986 ; 207 : 35-41.
Transducine : l'amplificateur. Connaissant la vitesse de réaction de la phosphodiestérase et sachant qu'un seul photon provoque l'hydrolyse de $10^{5}$ molécules de GMPc, il est possible de déduire qu'un photon active quelques centaines de molécules. Il a été remarqué que l'amplification nécessite l'action du GTP, ou de son analogue structural non hydrolysable, le GppNHp, donc l'interaction d'une protéine liant le GTP et se fixant à la rhodopsine photolysée sous l'action de la lumière [11]. Le transducteur a été purifié et son mécanisme d'action élucidé. Il s'agit d'une protéine fixant le GDP, et alternativement le GTP, dénommée transducine $(\mathrm{T})$ par Stryer en 1981 et constituée de 3 sousunités : alpha (39000 de poids moléculaire) possédant l'activité GTP-ase, bêta (35000) et gamma (8 000). A l'obscurité, la transducine fixe du GDP et est liée relativement fortement aux membranes discales dont elle est extractible par un choc hypotonique. A l'éclairement, la molécule entière se fixe sur l'extrémité C-terminale de la métarhodopsine II $\left({ }^{*} \mathrm{R}\right)$ par diffusion latérale dans la face cytoplasmique du disque. L'échange, au niveau de la sous-unité alpha (T $\alpha)$ du GDP fixé par du GTP, se produit en environ 1 milliseconde, ce qui contribue à dissocier le complexe car l'affinité de GTP-T pour ${ }^{*} \mathrm{R}$ chute : les sousunités GTP-T $\alpha$ et T $\beta \gamma$ se séparent et deviennent libres dans le cytoplasme pour activer la phosphodiestérase. La cinétique de dissociation de la transducine est conforme au niveau d'amplification (500 molécules environ) et au temps de latence de fermeture des canaux sodium (1 à 2 secondes) prédits.

La phosphodiestérase GMPc dépendante : l'effecteur. La phosphodiestérase a été purifiée en 1979 à partir de segments externes de bøuf [12]. Il s'agit d'un complexe de trois sous-unités : alpha (88 000 de poids moléculaire), bêta $(84000)$ et gamma (11 000), très peu actif dans l'obscurité. Des données de protéolyse ménagée et de reconstitution ont montré que la sous-unité $\gamma$ inhibe l'activité enzymatique et que l'activation de l'enzyme résulte de l'interaction de cette sous-unité $\gamma$ de la phosphodiestérase et de la sous-unité $\alpha$ de la transducine, ayant fixé du GTP ou du GppNHp, selon des modalités encore inconnues. L'activation cesse dès que la sous-unité $\alpha$ hydrolyse le GTP, redevenant ainsi capable de se réassocier avec $\mathrm{T} \beta \gamma$, puis la transducine totale se réassocie à la métarhodopsine II. Chaque photon induit environ 500 cycles d'activité GTP-ase. De plus, la phosphodiestérase possède, outre son site catalytique, des sites de fixation de haute affinité pour le GMPc, sites dont la saturation varie à l'inverse de l'activité catalytique. Ces sites pourraient ainsi constituer un réservoir de stockage de GMPc, pouvant le libérer rapidement et contribuer à restaurer l'état basal à l'obscurité. Fibroblast growth factor acide : un modulateur? Notre laboratoire, en collaboration avec celui d'Yves Courtois à Paris, vient de mettre récemment en évidence la fixation du Fibroblast growth factor (FGF) acide sur les membranes discales sous l'effet de la lumière, effet majoré par le GTP. En revanche, le FGF acide est libéré de ces membranes sous l'action d'une kinase [13]. Le FGF induit sélectivement la phosphorylation de deux protéines de 130 et 150 $\mathrm{kD}$ qui pourraient être ses récepteurs dans le bâtonnet (Plouët $e t$ al., résultats non publiés). Il a été récemment montré dans une lignée fibroblastique [14] que la fixation du FGF sur son récepteur cellulaire induisait une mobilisation du calcium extra-cellulaire médiée par une G-protéine sans activation de l'hydrolyse du phosphatidylinositol 4,5 diphosphate*. Il est ainsi possible d'emettre l'hypothèse que FGF pourrait, dans les bâtonnets, contrôler le flux calcique en fonction de la lumière (Plouët et al., soumis pour publication).

\footnotetext{
- Voir nouvelle m/s $n^{\circ} 3$, vol. 3, p. 174.
} 
L'ensemble de ces réactions est schématisé dans la figure $2 A$ et $2 B$.

\section{La désensibilisation}

L'amplification du signal photonique, une fois obtenue, s'arrête, puis des phénomènes aboutissant à des effets biologiques opposés sont mis en jeu afin de restaurer l'état initial.

Rhodopsine kinase : phosphorylation du récepteur. La phosphorylation d'un récepteur semble être une étape efficace de désensibilisation quel que soit le ligand considéré. Il existe effectivement une kinase spécifique de la rhodopsine, inactive à l'obscurité, qui phosphoryle la rhodopsine sur neuf résidus sérine thréonine, situés à l'extrémité C-terminale, sur le versant cytoplasmique du disque. Il s'agit d'une protéine de 68000 de poids moléculaire, autophosphorylable. Cette kinase est soluble à l'obscurité et se fixe aux membranes discales sous l'effet de la lumière [15] au niveau du site de fixation de la transducine, avec qui elle interfere. La faible concentration de la rhodopsine kinase comparée à celle de la transducine explique que la phosphorylation ne survienne qu'après que de nombreux cycles d'activation de la transducine aient été accomplis. Protéine $48 \mathrm{~K} /$ antigène-S : l'inhibition de l'effecteur. H. Kuhn avait montré en 1978 qu'une protéine soluble du segment externe se fixe sur les membranes discales illuminées [15], puis que cette fixation s'effectue beaucoup plus efficacement sur la rhodopsine illuminée et phosphorylée. Le rôle physiologique de cette substance a été récemment mis en évidence : elle inhibe la phosphodiestérase GMPc dépendante par liaison au niveau du site de fixation de la GDPtransducine, sans action directe sur la phosphodiestérase ellemême [16]. Cette protéine est aussi un auto-antigène soluble induisant l'uvéo-rétinite expérimentale : l'antigène-S [17] (voir l'article de Y. de Kozak et al., dans ce numéro). La régulation allostérique au niveau de la rhodopsine $m / s n^{\circ} 4$ ool. 3, aoril 87 a été étayée par des données de séquençage partiel des gènes de la sous-unité $\alpha$ de la transducine et de l'antigène-S qui présentent une forte homologie, en particulier au niveau d'une séquence peptidique impliquée dans le site de liaison du GTP [18]. Par ailleurs nous avons montre, à l'aide d'un anticorps monoclonal anti-antigène- $S$ reconnaissant le site de liaison du GTP [19], que ces deux protéines présentent une réactivité immunochimique croisée. Fonctionnellement, l'antigène-S jouerait ainsi un rôle de sous-unité alpha d'une protéine inhibitrice liant le GTP dans la transduction visuelle*

Un autre groupe de chercheurs propose cependant un mécanisme different pour expliquer l'inactivation de la phosphodiestérase par la $48 \mathrm{~K}$ : il s'agirait d'une protéine liant l'ATP et inhibant directement la phosphodiestérase [20].

Protéine kinase $\mathbf{C}$ : un modulateur. Il avait été montré en 1984 que les segments externes de bâtonnets de vertébrés contiennent une activité de type protéine kinase C. L'hydrolyse du phosphatidylinositol 4,5 diphosphate laissait supposer l'existence de la formation de diacylglycérol activant à son tour une protéine kinase C. Cette enzyme a été récemment purifiée dans les segments externes de bâtonnets de bœuf et est capable de phosphoryler la rhodopsine in vitro en des sites identiques à ceux phosphorylés par la rhodopsine kinase [21]. Or, cette phosphorylation in vitro n'est cependant en rien affectée par la lumière, ce qui n'est pas en faveur d'un rôle dans la transduction visuelle. Le faible pourcentage de rhodopsine phosphorylée à distance de toute illumination chez le bœuf pourrait peutêtre constituer un reflet de son activité.

- Voir nouvelle m/s $n^{\circ} 10$, vol. 2, p. 583 . lant l'activité de l'adénylate cyclase.

*.* ADP-ribosylation : frxation sur une protcine d'une molécule d'ADP-ribose produite par hydrolyse enzymatique du NAD+, coenzyme des déshydrogénases.
Ces réactions sont schématisées dans la figure $2 C$.

\section{Vers l'unité de la machi- nerie transductionnelle}

Le mécanisme d'activation de la phosphodiestérase par la lumière ressemble à l'activation de l'adénylate cyclase par une hormone : le signal transmis par le récepteur est amplifié par une ronde de protéines fixant le GTP. Plusieurs séries d'arguments évoquent la similitude des systèmes.

Interchangeabilité des constituants. Des expériences de reconstitution in vitro ont permis de soupçonner l'identité partielle de ces 2 couples récepteur-amplificateur : par exemple la rhodopsine illuminée augmente l'activité GTPase de la sous-unité catalytique de la protéine $G$ inhibitrice $\left(\mathrm{Gi}^{* *}\right.$ et, dans un moindre degré, de la sous-unité catalytique de la protéine $\mathrm{G}$ activatrice $(\mathrm{Gs})^{* *}$. La substitution par les sous-unités $\alpha$ de la transducine des sous-unités $\alpha$ de Gs inhibe l'activité adénylate cyclase des plaquettes [22]. La transducine activée par l'analogue GppNHp stimule l'adénylate cyclase de membranes synaptiques de cortex de rat. Par ailleurs, des préparations semi-purifiées de rhodopsine kinase et de kinase du récepteur $\beta$-adrénergique se sont révélées actives sur les 2 récepteurs activés ; elles sont inactives sur les récepteurs non illuminés ou non occupés par un agoniste $\beta$-adrénergique [23].

Action des toxines. La sous-unité Gs $\alpha$ est ADP-ribosylée ${ }^{* * *}$ par la toxine du choléra sous la forme GTP-Gs ; la sous-unité Gi $\alpha$ est ADP-ribosylée par la toxine de Bordetella pertussis sous la forme GDP-Gi. En revanche, T $\alpha$ est ADP-ribosylable par les deux toxines : T $\alpha$-GTP par la toxine du choléra et T $\alpha$-GDP par la toxine de Bordetella pertussis.

Comparaison des structures. La purification, l'immunoréactivité croisée et surtout l'isolement et le séquençage des gènes codant pour ces différentes protéines ont confirmé cette unité. La rhodopsine 


\section{RÉFÉRENCES}

21. Kelleher DJ. Johnson GL. Phosphorylation of rhodopsin by protein kinase $\mathrm{C}$ in vitro. $J$ Biol Chem 1986 ; 261 : 4749-56.

22. Bockaert J, Deterre P, Pfister C, Guillon $\mathrm{G}$, Chabre M. Inhibition of hormonally regulated adenylate cyclase by the subunit of transducin. $E M B O J 1985 ; 6: 1413-7$.

23. Benovic JL, Mayor JrF, Somers RL Caron MG, Lefkowitz RJ. Light dependent phosphorylation of rhodopsin by $\beta$-adrenergic receptor kinase. Nature 1986 ; 321 : 869-72.

24. Baehr W, Applebury ML. Exploring visual transduction with recombinant DNA techniques. TINS 1986 ; 198-203.

25. Codina J, Stengel D, Woo SLC, Birnbaumer L. Subunits of the human liver Gs (Gi signal-transducing proteins) and those of bovine retinal rod cell transducin are identical. FEBS Lett 1986 ; 207 : 187-92.

26. Aguirre G, Farber D, Lolley K, Fletcher RT, Chader GJ. Rod-cone dysplasia in Irish setters : a defect in cyclic GMP metabolism in visual cells. Science 1978 ; 201 : 1133-4.

Figure 2. Schématisation des interactions protéiques lors des étapes do la transduction visuollo. $A$ : obscurité ; B : lumière ; C : désensibilisation. Le pigment visuel se présente successivement sous la forme rhodopsine (R), métarhodopsine "I $\left(R^{*}\right)$ et opsine phosphorylée $\left(R^{P}\right)$. Td : transducine; PDE: phosphodiestérase GMPc dépendante; RK : rhodopsine kinase; $48 K$ : protéine $48 K$ ou antigène-S; GC : guanylate cyclase ; $K c$ : protéine kinase $C$; FGF : facteur de croissance rétinien identique au "Fibroblast Growth Factor " acide. A. A l'obscurité, Td est libre dans le cytoplasme, PDE est inactive et le canal sodium est ouvert. B. L'illumination provoque la fixation de GDP-Td sur $R^{*}$. L'échange de GDP par GTP sur $T \alpha$ dissocie l'hétérotrimère : $T \alpha$ GTP se fixe sur la sous-unité y de la PDE qui devient activée. Le GMPc diminue transitoirement et le canal sodium se ferme. Le taux de calcium diminue, contribuant ensuite dे augmenter la synthèse de GMPc. Le GTP de $T \alpha$ est hydrolysé et la transducine se réassocie, initiant ainsi un nouveau cycle. RK phosphoryle R*. FGF se fixe sur les membranes discales. La concentration en IP3 augmente. C. La phosphorylation des membranes entraine la fixation de $48 K$ sur $R^{p}$ masquant ainsi le site de liaison pour GDP T $\alpha$. La PDE s'inactive. Le taux de GMPc augmente et permet la réouverture des canaux sodium. La concentration en calcium réaugmente. FGF est relargué des membranes.
(A)

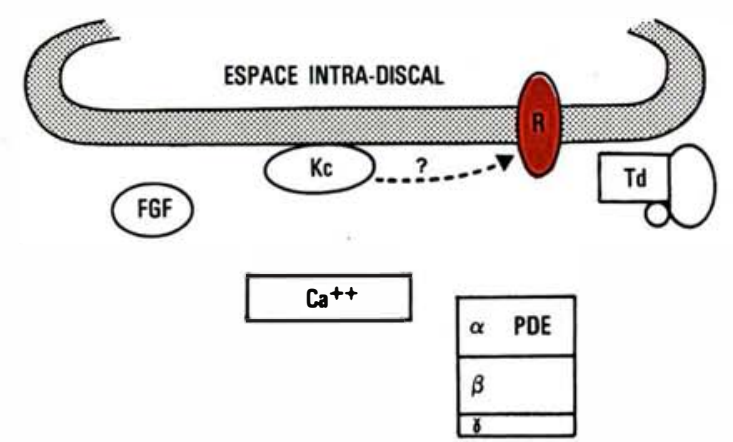

(B)

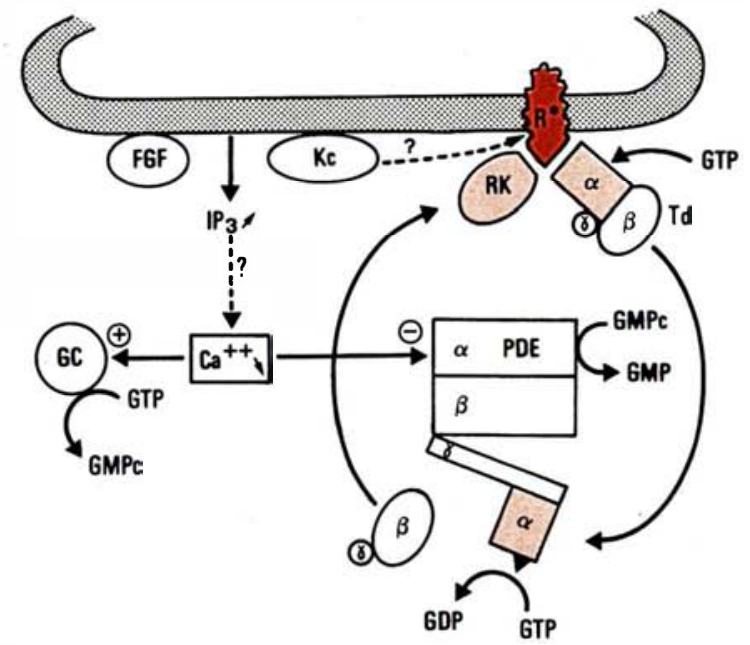

(C)

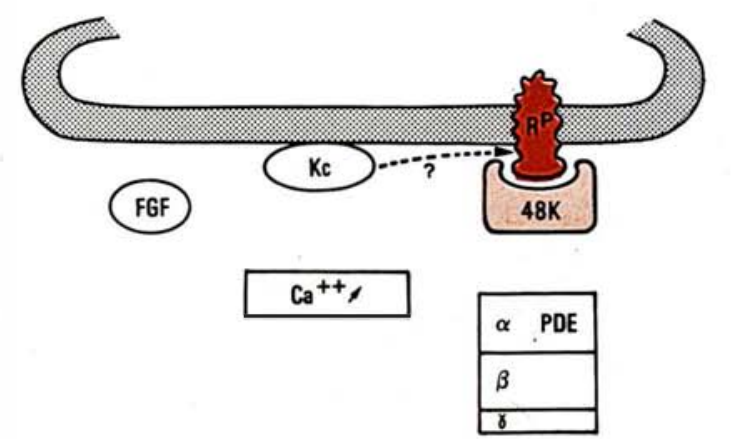

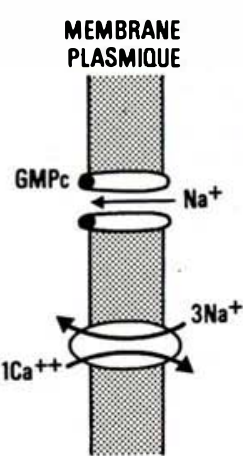

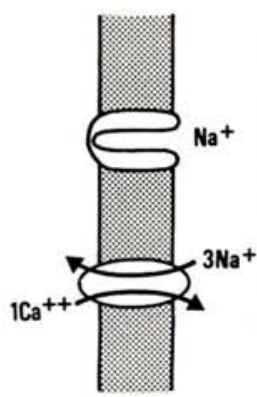


présente une homologie avec le récepteur bêta-adrénergique, en particulier au niveau de l'extrémité C-terminale riche en résidus sérine et thréonine où s'effectuent les phosphorylations par les deux kinases, ainsi qu'une homologie avec le récepteur muscarinique. Les gènes des différentes Gprotéines ont été isolés, ce qui a permis de les regrouper au sein d'une famille constituée de la transducine $\mathrm{T} \alpha$, d'une transducine spécifique des cônes, de Gs, de Gi, de Go (une G-protéine de fonction inconnue existant dans le cerveau), de $\mathrm{Gp}$ dans le placenta, de $G$ olf (spécifique de l'olfaction), de $p 21$ produit du gène ras, de EFTu le facteur d'élongation de Escherichia coli, enfin de la protéine $48 \mathrm{~K}$-antigène-S. L'examen comparé des séquences des gènes de la famille opsine et de la famille G-protéine a montré une zone de recouvrement entre les domaines 5-6 de l'opsine et le domaine GTPase des G-protéines. Ceci soulève la question de l'existence d'une protéine ancestrale absorbant la lumière et fixant, puis hydrolysant le GTP. Baehr et Applebury [24] proposent que la modification de la rhodopsine sous l'effet de la lumière démasquerait un site de liaison pour le complexe $\beta \gamma$, permettant la fixation transitoire de la transducine au niveau de ces sites de la rhodopsine activée (sous forme métarhodopsine II). L'échange du GDP lié à la sous-unité T $\alpha$ par du GTP entraînerait alors la dissociation entre $\mathrm{T} \alpha, \mathrm{T} \beta \gamma$ et la rhodopsine, initiant ainsi la cascade de réactions décrites plus haut. $\mathrm{Par}$ ailleurs, les sous-unités $\beta$ de Gs, $\mathrm{Gi}$ et transducine sont identiques [25], les sous-unités $\gamma$ en revanche sont différentes.

\section{Pathologie de la transduction visuelle?}

Il n'existe pas actuellement de réelle corrélation entre une anomalie d'un constituant de la transduction visuelle et une maladie se développant spontanément. Tout au plus peut-on évoquer deux axes de recherche.

$\mathrm{m} / \mathrm{s} n^{\circ} 4$ ool. 3, aoril 87
Rétinite pigmentaire. Il s'agit d'une dystrophie rétinienne génétiquement transmise, d'évolution lente et inéluctable vers la dégénérescence des photorécepteurs. Dans des dystrophies similaires du rat, de la souris et du chien, il existe une corrélation entre la dystrophie et l'accumulation de GMPc [26] dans le segment externe. Il semble qu'il puisse coexister au moins 2 anomalies métaboliques : la présence d'une phosphodiestérase inactive et d'une phosphatase hyperactive déphosphorylant la rhodopsine phosphorylée. Celle-ci ne fixant plus la $48 \mathrm{~K}$, ne permettrait plus une régulation de la phosphodiestérase. Il pourrait aussi exister des anomalies du fonctionnement des modulateurs de la transduction.

Uvéo-rétinite auto-immune. Ce paragraphe est traité dans l'article de Y. de Kozak et al., dans ce numéro. La maladie expérimentale peut être induite directement chez l'animal par au moins deux antigènes différents impliqués dans la transduction : la rhodopsine et l'antigène-S. D'autre part, des arguments indirects suggèrent que la rhodopsine kinase et la transducine seraient également des auto-antigènes (résultats non publiés). Les éventuelles corrélations avec d'autres maladies autoimmunes intéressant des structures homologues (auto-immunité contre le récepteur muscarinique dans la myasthénie, par exemple) restent spéculatives.

En conclusion, le segment externe du bâtonnet se présente comme une entité physiologique entièrement dévolue à une fonction différenciée univoque : la discrimination et l'adaptation à la lumière. Une telle spécialisation est probablement unique dans un tissu, ce qui fait de cette unité fonctionnelle qu'est le disque un matériel d'excellente qualité pour l'étude de la transmission et de l'intégration des communications intercellulaires. Elle pourrait constituer également un modèle général d'étude de la physiopathologie des dysfonctionnements de la communication intercellulaire

\section{Summary}

The process of light discrimination takes place in the outer segment of the photoreceptor cells of the retina. This highly specialized subfraction can be obtained in relatively large amounts in the form of undamaged and purified material : the disks. The second messenger involved in the conversion of light into electrical current has been identified as cyclic guanylate monophosphate. The mechanisms of amplification of the light signal have many similarities with those implicated in the amplification of hormonal signals. It seems likely that study of visual transduction may lead to a better understanding of the pathophysiology of hormonal transduction.

\section{TIRÉS A PART}

J. Plouẽt : Inserm U. 86, laboratoire d'immunopathologie de l'ail, centre des Cordeliers, 15, rue de l'École de Médecine, 75270 Paris Cedex 06. 